\title{
Influence of the Fermi surface geometry on the Josephson effect between iron-pnictide and conventional superconductors
}

\author{
A. A. Kalenyuk $\odot,{ }^{1,2,3}$ E. A. Borodianskyi, ${ }^{1}$ A. A. Kordyuk, ${ }^{2,3}$ and V. M. Krasnov ${ }^{1,4, *}$ \\ ${ }^{1}$ Department of Physics, Stockholm University, AlbaNova University Center, SE-10691 Stockholm, Sweden \\ ${ }^{2}$ Institute of Metal Physics of National Academy of Sciences of Ukraine, 03142 Kyiv, Ukraine \\ ${ }^{3}$ Kyiv Academic University, 03142 Kyiv, Ukraine \\ ${ }^{4}$ Moscow Institute of Physics and Technology, State University, 141700 Dolgoprudny, Russia
}

(Received 11 February 2021; revised 20 April 2021; accepted 27 May 2021; published 10 June 2021)

\begin{abstract}
We study hybrid Josephson junctions between a multiband $\mathrm{Ba}_{1-x} \mathrm{Na}_{x} \mathrm{Fe}_{2} \mathrm{As}_{2}$ iron-pnictide and $\mathrm{Nb}$. We observe that the insertion of a $\mathrm{Cu}$ interlayer in such junctions leads to a dramatic enhancement of the $I_{c} R_{n}$ product, despite the weaker proximity-induced superconductivity of $\mathrm{Cu}$. This counterintuitive phenomenon is attributed to the differences in Fermi surface geometries of $\mathrm{Nb}$ and $\mathrm{Cu}$, which affect the selectivity of tunneling in sign-reversal $s_{ \pm}$bands of pnictide. Our results indicate that the sensitivity to Fermi surface geometries provides a new tool for phase-sensitive studies and paves the way to conscious Fermi surface engineering of pnictide junctions.
\end{abstract}

DOI: 10.1103/PhysRevB.103.214507

\section{INTRODUCTION}

The electronic structure of superconductors is usually quite complicated, even for low- $T_{c}$ materials, such as the transition metal $\mathrm{Nb}$. Nevertheless, a simple description of the Josephson effect, which does not take into account complex Fermi surface (FS) geometry, works remarkably well for conventional superconductors $[1,2]$. This happens because the probabilities of electron and Cooper pair tunneling are similar [3]. Together with the momentum-independent $s$-wave energy gap, $\Delta$, it leads to the inverse relationship between the normal resistance, $R_{n}$, and the critical current, $I_{c}$. Thus, the $I_{c} R_{n}$ product becomes a universal function of $\Delta$, independent of the FS geometry [4].

This universality, however, breaks for unconventional multiband superconductors. A particularly drastic deviation should happen in the case of the sign-reversal order parameter [5-8]. This occurs in cuprate- and iron-based superconductors, which are believed to have $d$-wave $[9,10]$ and $s_{ \pm}[11-18]$ symmetries, respectively. In this case, $I_{c}$ depends on the gap values in each band, and the $I_{c} R_{n}$ is band structure sensitive and not universal [5-8] (more discussion can be found in Appendix E).

In this work we study Josephson junctions (JJs) between an iron-pnictide $\mathrm{Ba}_{1-x} \mathrm{Na}_{x} \mathrm{Fe}_{2} \mathrm{As}_{2}$ (BNFA) single crystal and conventional low- $T_{c}$ superconductors made of either a $\mathrm{Nb}$ or a $\mathrm{Cu} / \mathrm{Nb}$ bilayer. Both types of JJs exhibit clean and clear

\footnotetext{
*vladimir.krasnov@fysik.su.se
}

Published by the American Physical Society under the terms of the Creative Commons Attribution 4.0 International license. Further distribution of this work must maintain attribution to the author(s) and the published article's title, journal citation, and DOI. Funded by Bibsam.
Josephson phenomena. However, JJs with a $\mathrm{Cu}$ interlayer exhibit an almost two orders of magnitude larger $I_{c} R_{n}$, despite the weaker proximity-induced superconductivity of $\mathrm{Cu}$. This counterintuitive result is explained by the difference in FS geometries of $\mathrm{Nb}$ and $\mathrm{Cu}$, which affects the selectivity of tunneling in different sign-reversal $s \pm$ bands of BNFA. Our data indicate that the sign reversal occurs between central and corner bands of BNFA, which, although anticipated [12-15], has not yet been proven experimentally. Therefore, the reported strong interlayer dependence of the Josephson effect provides a new phase-sensitive tool for fundamental studies of unconventional superconductivity and opens the possibility of conscious Fermi surface engineering of pnictide JJs.

\section{EXPERIMENTAL PROCEDURE}

Figure 1(a) represents a scanning electron microscope (SEM) image of the BNFA- $\mathrm{Cu} / \mathrm{Nb}$ sample. Each sample contains six JJs made on a freshly cleaved BNFA crystal. Micrometer-sized JJs are formed in the overlap area between a window-line opening in the $\mathrm{SiO}_{2}$ isolation layer and the top contact electrodes, as shown in the sketch in Fig. 1(b). As electrodes we use either pure $\mathrm{Nb}$ or a $\mathrm{Cu}(15 \mathrm{~nm}) / \mathrm{Nb}$ bilayer deposited by magnetron sputtering in a single cycle without breaking the vacuum. Details of the sample fabrication and experimental setup and a list of JJ parameters can be found in Appendixes A-C.

The multiterminal configuration of our samples allows simultaneous measurements of junction and crystal characteristics $[18,19]$. The blue line in Fig. 1(c) shows the in-plane resistive transition of BNFA. At $T \sim 150 \mathrm{~K}$ there is a kink in $R(T)$, corresponding to structural and spin-density-wave transitions $[13,15]$. The superconducting transition occurs at $T_{c}(\mathrm{BNFA}) \simeq 30 \mathrm{~K}$, indicating a moderately underdoped state of BNFA. The red line in Fig. 1(c) shows $R(T)$ of a JJ. It has 

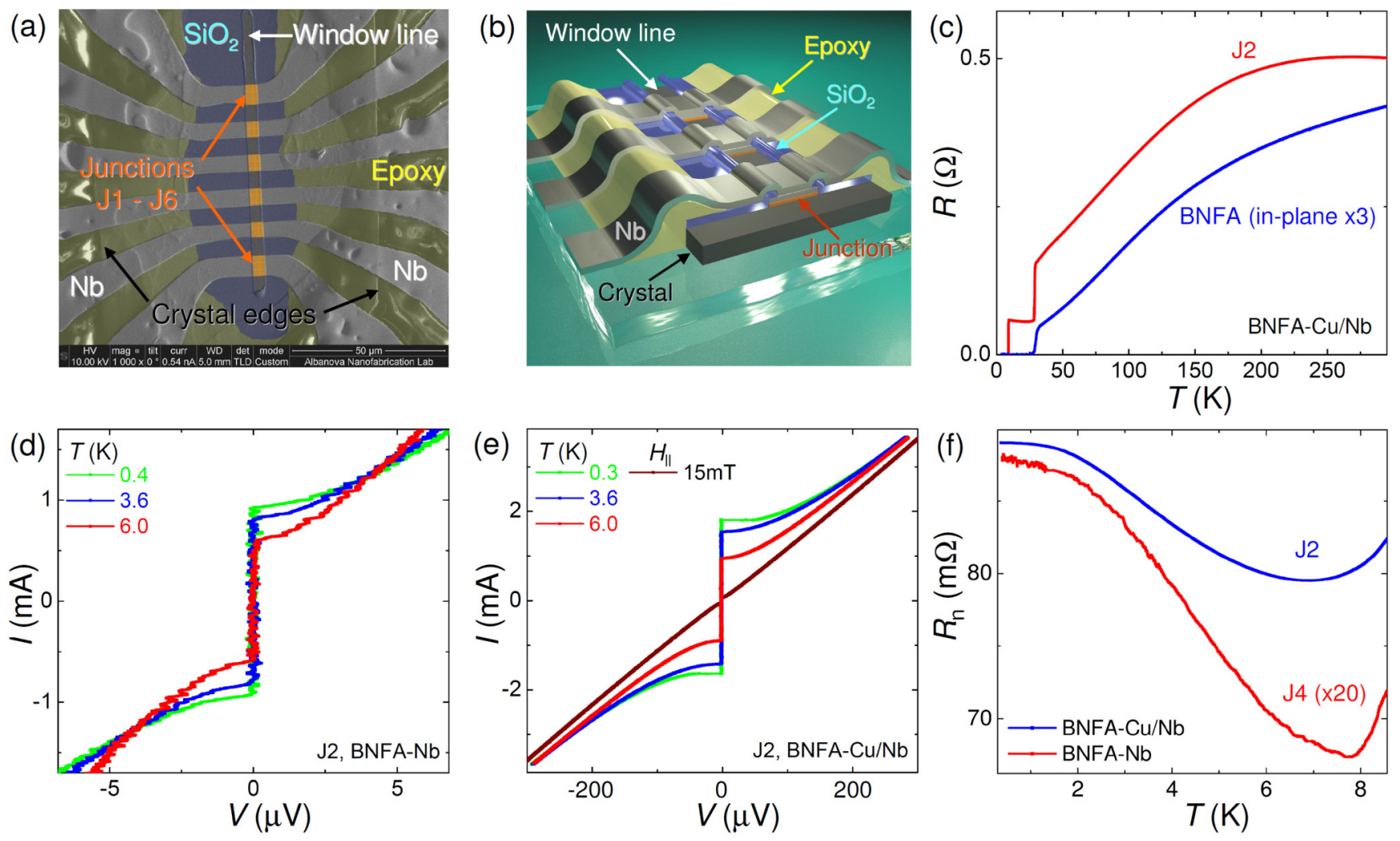

FIG. 1. (a) SEM image of a BNFA-Cu/Nb sample (painted). (b) Sketch of the sample. Junctions are formed at a freshly cleaved surface of the crystal in the area at the overlap between a window-line opening in $\mathrm{SiO}_{2}$ and the top $\mathrm{Nb}$ electrodes. (c) Resistive transitions of a junction (J2; red line) and BNFA crystal (blue line) for the BNFA-Cu/Nb sample. (d), (e) $I-V$ curves at different temperatures and $H=0$ for junctions at BNFA-Nb (d) and BNFA-Cu/Nb (e) samples. The wine-colored curve in (e) represents $I-V$ at $T \simeq 0.3 \mathrm{~K}$ and $H_{\|}=15 \mathrm{mT}$. It demonstrates the complete suppression of $I_{c}$ by a modest in-plane magnetic field. (f) Temperature dependencies of normal resistances for BNFA-Nb (red line) and BNFA-Cu/Nb (blue line) junctions.

two steps, the first at $T_{c}(\mathrm{BNFA})$ and the second at $T_{c}(\mathrm{Nb}) \simeq$ $9 \mathrm{~K}$.

Figure 1 shows the current-voltage $(I-V)$ characteristics at different $T$ 's for BNFA-Nb [Fig. 1(d)] and BNFA-Cu/Nb [Fig. 1(e)] JJs. The $I-V$ 's have the shape typical of resistively shunted JJs $[1,2]$ with a well-defined $I_{c}$ and $R_{n}$. The green, blue, and red curves are measured at zero field. The winecolored line in Fig. 1(e) shows the $I-V$ at an in-plane magnetic field of $B_{\|}=15 \mathrm{mT}$. It is shown that $I_{c}$ is completely suppressed by a small parallel field, much smaller than $H_{c 2}$ of BNFA [19,20] and $\mathrm{Nb}$ [21]. Therefore, in such fields we can accurately measure the $R_{n}(T)$ dependencies of JJs, shown in Fig. 1(f). The modest upturn of $R_{n}$ with decreasing $T$ is typical for $c$-axis characteristics of high- $T_{c}$ superconductors, commonly associated with the pseudogap phenomenon [22].

\section{RESULTS}

Suppression of $I_{c}$ by a small parallel field is caused by flux quantization. Figure 2(a) shows $I_{c}\left(H_{\|}\right)$modulations at different $T$ 's for a BNFA-Cu/Nb JJ. Figure 2(b) represents modulation patterns for BNFA-Nb (blue line) and BNFA- $\mathrm{Cu} / \mathrm{Nb}$ (red line) JJs. Both exhibit clear Fraunhofer modulation, depicted by the black line. This is a figure of merit, indicating good uniformity of JJs [1,2].
Figure 2(c) shows $I-V$ curves of a BNFA-Cu/Nb JJ without (black line) and with (red line) electromagnetic radiation at $f \simeq 74 \mathrm{GHz}$. A primary Shapiro step is seen at $V_{1}=h f / 2 e$. Figure 2(d) shows the normalized differential conductance for this $I-V$ as a function of $V_{1} / V$. It reveals numerous subharmonic Shapiro steps. This indicates a nonsinusoidal current-phase relation [23], which is indeed anticipated for $s-s_{ \pm} \mathrm{JJs}[7,8]$. On the other hand, it may also be caused by the proximity effect in the $\mathrm{Cu} / \mathrm{Nb}$ bilayer [24]. Thus, our JJs exhibit clean and clear dc- and ac-Josephson effects. The high quality and the good reproducibility of JJs (see Appendix C) allow a fundamental analysis of superconductivity in BNFA (as opposed to interface defects).

Figure 2 shows the $T$ dependencies of the critical current density $J_{c}$ [Fig. 2(e)] and the $I_{c} R_{n}$ product [Fig. 2(f)] for both types of JJs. Despite their similarities in behavior and the same BNFA crystal and fabrication procedure, they exhibit largely different $I_{c} R_{n}$ values. BNFA-Nb JJs have a very small $I_{c} R_{n} \simeq$ $3 \mu \mathrm{V}$ [18], much smaller than $\Delta / e>1 \mathrm{mV}$ in both superconductors, while for BNFA-Cu/Nb JJs $I_{c} R_{n} \simeq 200 \mu \mathrm{V}$. The increase in $I_{c} R_{n}$ in BNFA-Cu/Nb JJs is associated with the increase in $R_{n}$ (see Appendix C). The latter indicates that the interface transparency, $\beta$, between BNFA and $\mathrm{Cu}$ is reduced compared to that of BNFA-Nb. Yet, as mentioned in Sec. I, this does not explain the increase in $I_{c} R_{n}$ because usually $I_{c} \propto 1 / R_{n}$ and $I_{c} R_{n}$ is independent of $\beta$. Cu is a normal metal. 

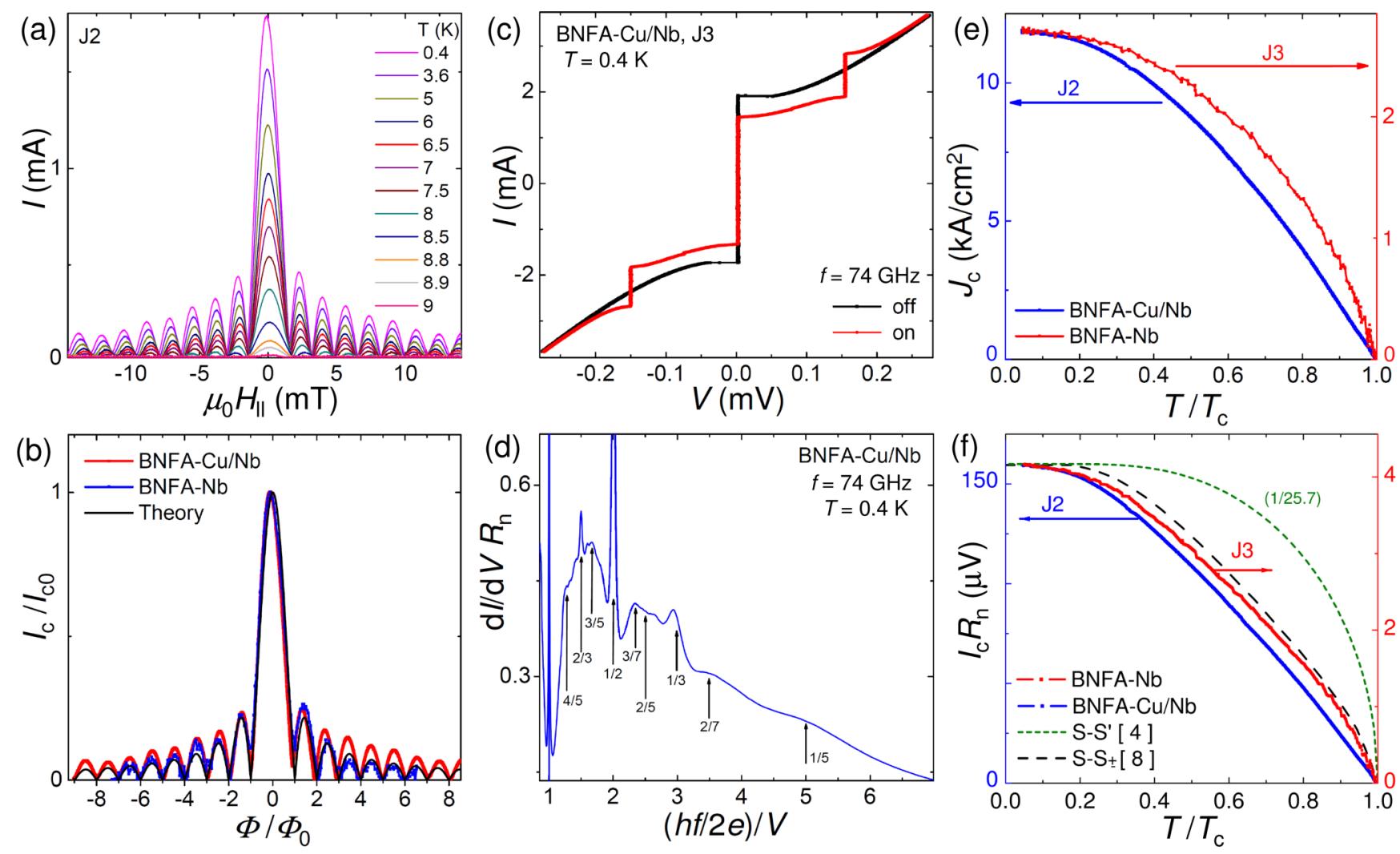

FIG. 2. (a) Modulation of the critical current versus the in-plane magnetic field for a BNFA-Cu/Nb junction at different temperatures. (b) Comparison of $I_{c}$ versus flux modulation patterns of BNFA- $\mathrm{Cu} / \mathrm{Nb}$ (red line) and BNFA-Nb (blue line) and the Fraunhofer pattern (black line). (c) $I-V$ characteristics of a BNFA-Cu/Nb junction with (red line) and without (black line) microwave radiation. The primary Shapiro step is clearly shown. (d) Differential conductance versus inverse voltage, demonstrating the presence of subharmonic Shapiro steps. (e) Temperature dependencies of critical current densities of BNFA-Cu/Nb (blue line; left scale) and BNFA-Nb (red line; right scale) junctions. (f) Temperature dependencies of $I_{c} R_{n}$ products for the same junctions. Note the big difference in $I_{c} R_{n}$ values. The dotted black line represents a normalized theoretical curve for conventional $s$-wave superconductors [4]. The dashed black line shows a simulated dependence for an $s$ - $s_{ \pm}$ junction from Ref. [8].

The superconducting order parameter in $\mathrm{Cu}$ is induced via the proximity effect with $\mathrm{Nb}$ and is smaller than in $\mathrm{Nb}$ [25]. For such SINS JJs made of conventional $s$-wave superconductors, the proximity effect always leads to a reduction of $I_{c} R_{n}$ [26]. This is opposite to our observation. The reported remarkable influence of the $\mathrm{Cu}$ interlayer is the key new observation of this work.

\section{DISCUSSION}

To clarify the role of the $\mathrm{Cu}$ interlayer, we consider the FS geometries of the involved metals. Figure 3 shows the calculated Fermi surfaces for $\mathrm{Cu}$ [Fig. 3(a)], Nb [Fig. 3(b)] [27,28], and BNFA [Fig. 3(c)] [29]. The FS of $\mathrm{Cu}$ is simple quasispherical. $\mathrm{Nb}$ has a very complex FS with many small pockets spread over the Brillouin zone (BZ). BNFA has two bunches of FS sheets (colloquially referred to as two bands), three large quasicylinders in the center, and propellerlike FSs at the corners of the BZ [30,31].

Electron tunneling usually conserves the in-plain momentum $\mathbf{k}_{\|}=\left(k_{x}, k_{y}\right)$. Therefore, the current can be written as

$$
I \propto \oint T_{12}^{2} A_{1}\left(k_{z 1}, \mathbf{k}_{\|}\right) A_{2}\left(k_{z 2}, \mathbf{k}_{\|}\right)\left(f_{1}-f_{2}\right) d \mathbf{k}_{\|} d k_{z 1} d k_{z 2}
$$

where $T_{12}$ is the matrix element between initial $\left(k_{z 1}, k_{x}, k_{y}\right)$ and final $\left(k_{z 2}, k_{x}, k_{y}\right)$ states, $A_{1,2}$ are spectral functions [momentum-dependent density of states (DoS)], and $f_{1,2}$ are distribution functions. The key FS-dependent factors are DoS projections on the junction plane at Fermi energies,

$$
N_{i}\left(\mathbf{k}_{\|}\right)=\int A_{i}\left(k_{z i}, \mathbf{k}_{\|}\right) d k_{z i} \quad(i=1,2) .
$$

Figures 3(d) and 3(e) show such DoS projections for $\mathrm{Cu}$ and $\mathrm{Nb}$. The corresponding projection for BNFA is fairly similar to the pattern in Fig. 3(i).

For comparison with experiment we should take into account the polycrystalline structure of our films. To account for the random crystalline orientation, we average out the DoS with respect to rotation of the $k_{x, y}$ axes. Figures 3(f) and 3(g) show the thus obtained $\left\langle N_{i}\left(k_{x}, k_{y}\right)\right\rangle$ for polycrystalline $\mathrm{Cu}$ and $\mathrm{Nb}$. The key difference is that the DoS of $\mathrm{Cu}$ maintains a circular shape, while the DoS of $\mathrm{Nb}$ acquires a fairly uniform distribution over the BZ (see also Appendix D).

Figure 3 shows products of DoS projections $\left\langle N_{\mathrm{Cu}}\left(\mathrm{k}_{\mathrm{x}}, \mathrm{k}_{\mathrm{y}}\right)\right\rangle \mathrm{N}_{\mathrm{BNFA}}\left(\mathrm{k}_{\mathrm{x}}, \mathrm{k}_{\mathrm{y}}\right)$ for BNFA-Cu/Nb [Fig. 3(h)] and $\left\langle N_{\mathrm{Nb}}\left(k_{x}, k_{y}\right)\right\rangle N_{\mathrm{BNFA}}\left(k_{x}, k_{y}\right)$ for BNFA-Nb [Fig. 3(i)] JJs. They give hints about the current flow in BNFA bands. For BNFA-Nb JJs both BNFA bands participate approximately 

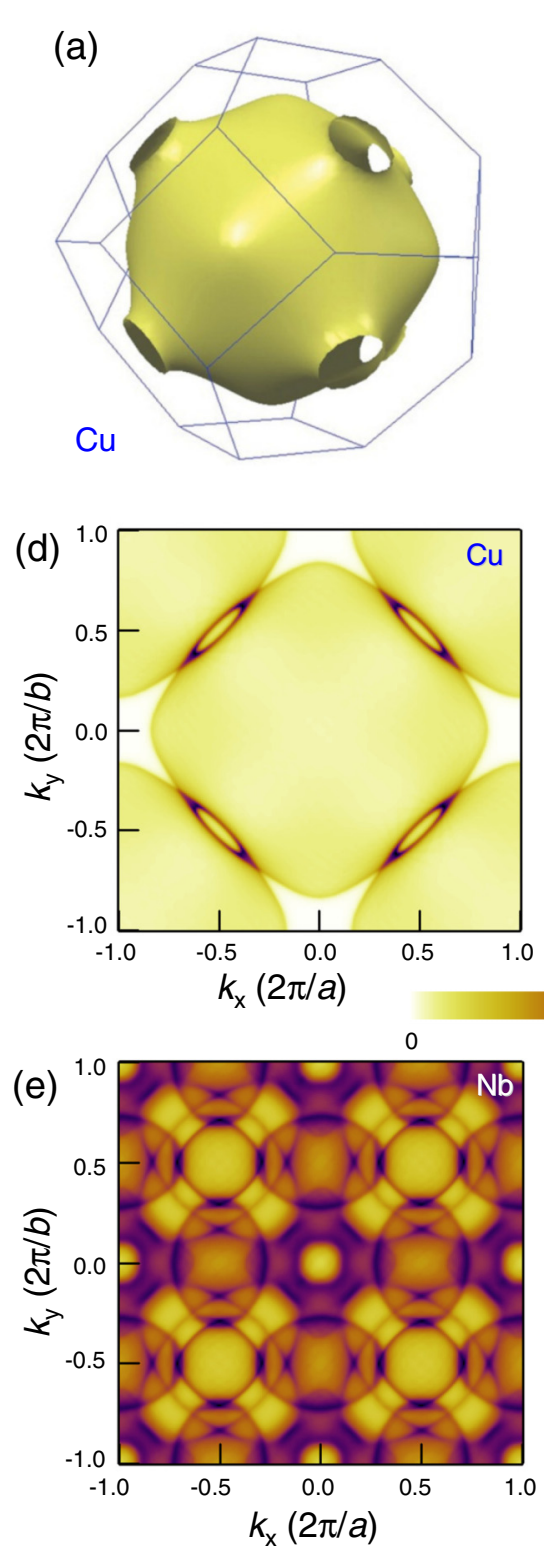

(b)
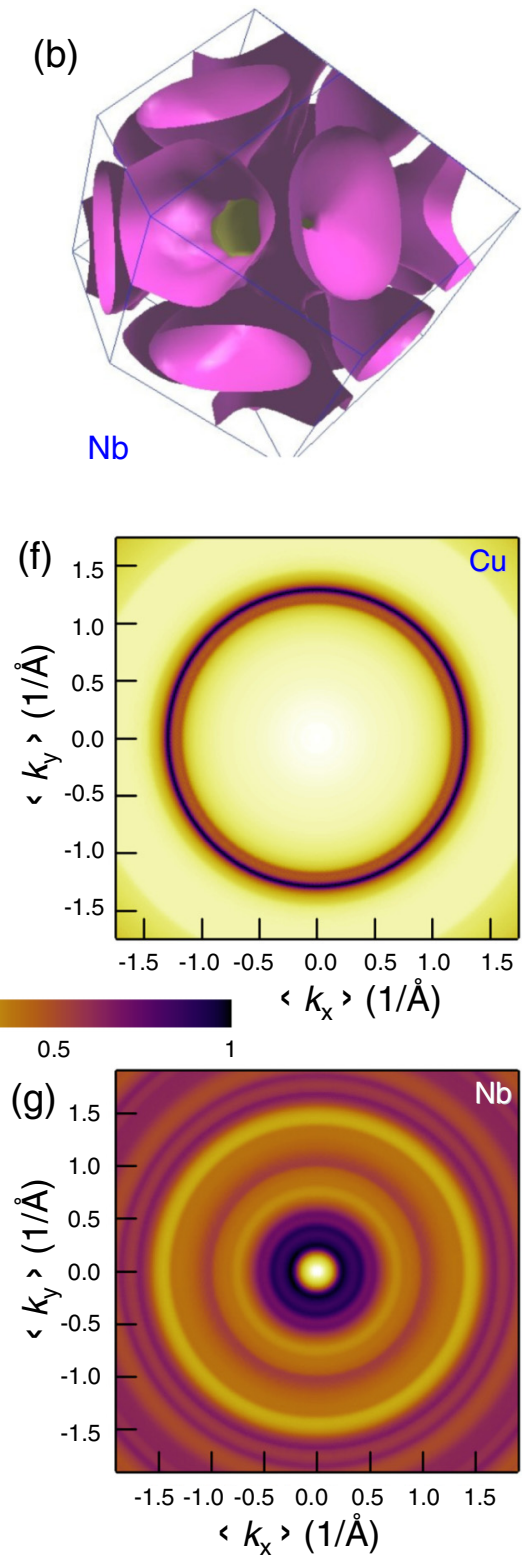

(c)
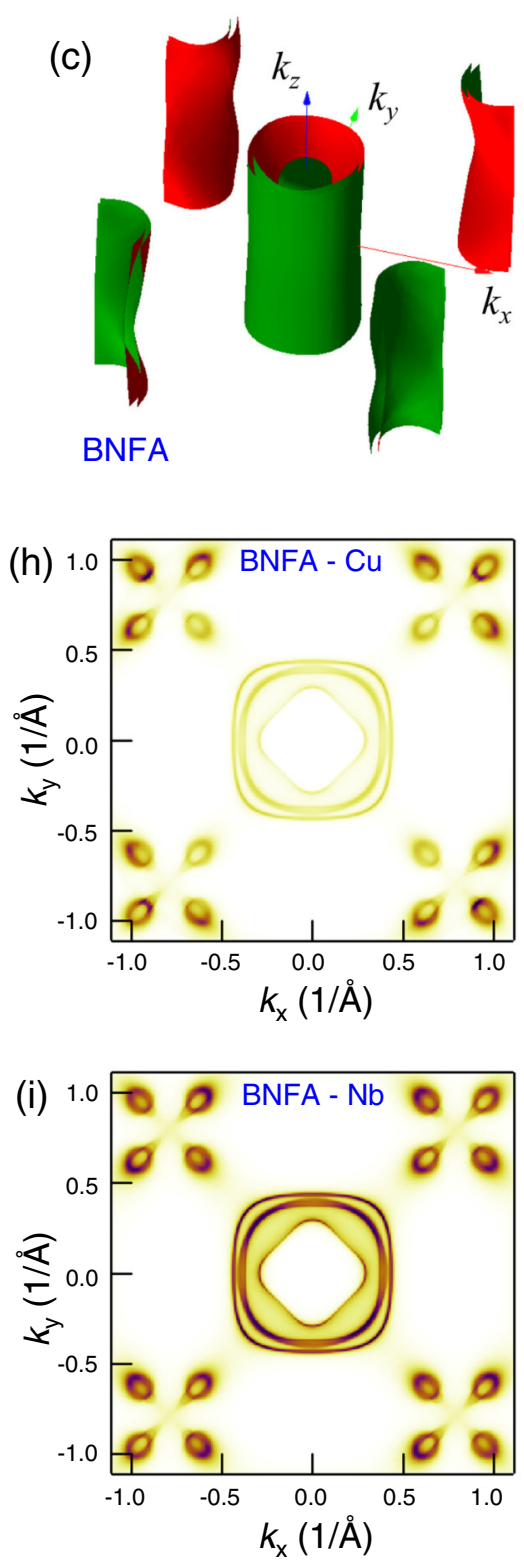

FIG. 3. Fermi surface topologies in the first Brillouin zone for (a) $\mathrm{Cu}$, (b) $\mathrm{Nb}$, and (c) BNFA. $k_{z}$-integrated projections of densities of states on the (001) plane for (d) $\mathrm{Cu}$ and (e) $\mathrm{Nb}$. Similar averaged DoS projections for polycrystalline (f) $\mathrm{Cu}$ and (g) $\mathrm{Nb}$ films. Products of DoS projections of monocrystalline BNFA with polycrystalline (h) $\mathrm{Cu}$ and (i) $\mathrm{Nb}$. It can be seen that for BNFA-Cu junctions (h) the tunneling current flows predominantly into the corner propellerlike bands of BNFA. On the other hand, for BNFA-Nb junctions (i) it is distributed relatively uniformly between central and corner bands.

equally due to the fairly uniform distribution of the $\operatorname{DoS}(\mathrm{Nb})$ in the BZ [Fig. 3(g)]. On the contrary, the highly nonuniform, circular-shaped $\operatorname{DoS}(\mathrm{Cu})$ [Fig. 3(f)] supports tunneling into corner bands and suppresses the current in the central bands.

Cooper pair tunneling depends both on the DoS and on the amplitudes and signs of $\Delta$ in each band. In Appendix E we present a simple model for calculation of $I_{c} R_{n}$ for JJs with a two-band superconductor. It is shown that in the signconserving $s_{++}$case the $I_{c} R_{n}$ is only slightly dependent on the FS geometry. On the contrary, in the sign-reversal $s_{ \pm}$ case the $I_{c} R_{n}(T)$ strongly depends on the FS geometries, with spectacular variations taking place close to the cancellation point $I_{c}=0$.
Our data are consistent with this scenario: the BNFA$\mathrm{Nb}$ JJs with $I_{c} R_{n}(0) \simeq 3 \mu \mathrm{V} \ll|\Delta| / e$ are very close to the cancellation point [18], implying that Cooper pair tunneling occurs at equal rates into both $s_{ \pm}$bands. This occurs due to the even distribution of the DoS(Nb), as shown in Figs. 3(g) and 3(i). The addition of a $\mathrm{Cu}$ interlayer with a very different FS geometry blocks tunneling in the central bands of BNFA [see Fig. 3(h)] and removes the cancellation. This leads to a dramatic enhancement of $I_{c} R_{n}$, accompanied by an increase in $R_{n}$ (for more details see the Appendix E).

We argue that the strong FS-geometry dependence of the Josephson effect provides a new phase-sensitive tool for investigation of unconventional superconductivity. For 
example, while the near-cancellation of $I_{c} R_{n}$ in BNFA-Nb JJs is evidence of $s_{ \pm}$symmetry in BNFA [18], the dramatic enhancement of $I_{c} R_{n}$ in NBFA-Cu/Nb JJs points out that a sign reversal occurs between the central and the corner bands of BNFA. This, although anticipated [12-15], has not been proven experimentally. FS-dependent effects can probably explain the reported spread of $I_{c} R_{n}$ in pnictide JJs [8,18,32-34]. This tool can also be applied to other unconventional sign-reversal superconductors, including cuprates, which are believed to have a dominant $d$-wave order parameter $[9,10]$. However, recent experiments revealed a more complex FS geometry of cuprates [35-37], with coexisting small pockets and large barrels. The gap symmetry in such a multiband case is not established and could be examined using FS-selective tuneling.

\section{CONCLUSIONS}

To conclude, we have fabricated and studied high-quality pnictide-Nb Josephson junctions. We observed that the insertion of a thin $\mathrm{Cu}$ interlayer increases the $I_{c} R_{n}$ product by almost two orders of magnitude, opposite to expectations for proximity-coupled junctions. This counterintuitive phenomenon is explained by the strong sensitivity of $s-s_{ \pm}$ junctions to Fermi surface geometries of involved materials. We argue that Fermi surface engineering of such junctions (choice of interlayer material, crystalline structure, orientation, composition, doping states, etc.) could provide a new tool for phase-sensitive studies of unconventional superconductivity and can be used for optimization and conscious engineering of Josephson junctions.

\section{ACKNOWLEDGMENTS}

The work was supported by the National Research Foundation of Ukraine (Project 2020.02/0408) and the Russian Science Foundation (Grant No. 19-19-00594). The paper was written during V.M.K.'s sabbatical at Moscow Institute of Physics and Technology. We are grateful to A. N. Yaresko for providing the results of DTF calculations, V. V. Zabolotnyy for help with FS visualization, and S. Aswartham, S. Wurmehl, and B. Büchner for providing BNFA crystals.

\section{APPENDIX A: SAMPLE FABRICATION}

The BNFA-Nb and BNFA- $\mathrm{Cu} / \mathrm{Nb}$ junctions were made as follows. An original large BNFA crystal $\left(\sim 1 \times 1 \mathrm{~mm}^{2}\right)$ was cut and cleaved into small and thin flakes $\left(\sim 150 \times 50 \mu \mathrm{m}^{2}\right.$ in the $a b$ plane and a few $\mu \mathrm{m}$ in the $c$-axis direction). A proper flake was glued with epoxy between two sapphire substrates $\left(5 \times 5 \mathrm{~mm}^{2}\right)$ and cleaved along the $a b$ plane (the easy cleavage plane), opening a fresh $c$-axis-oriented surface. Immediately after this a thin $\mathrm{Nb}(\sim 70 \mathrm{~nm})$ or double-layer $\mathrm{Cu}(\sim 15 \mathrm{~nm}) / \mathrm{Nb}(\sim 70 \mathrm{~nm})$ film was deposited by dc + rf magnetron sputtering. A narrow, $\sim 6-\mu \mathrm{m}$, line was patterned using photolithography and reactive ion etching with cryogenic Ar-ion etching to remove the $\mathrm{Cu}$ layer. Next, an insulating $\mathrm{SiO}_{2} \sim 80 \mathrm{~nm}$ layer was deposited and a window to the line was opened by the liftoff process. Additional insulation around the line and crystal corners was made with the epoxy glue. Finally, the second $\mathrm{Nb}$ layer, $\sim 130 \mathrm{~nm}$, was deposited
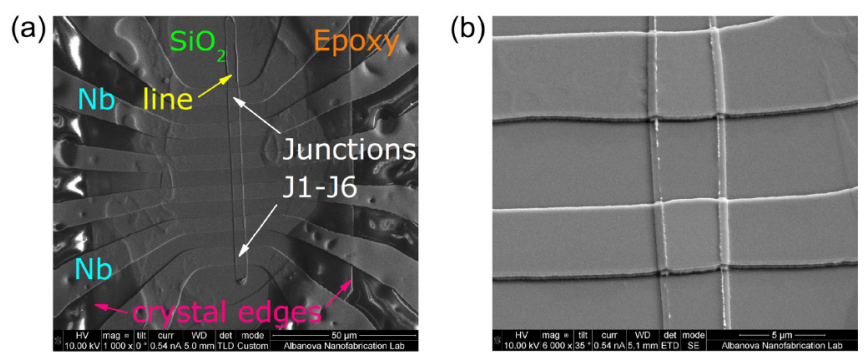

FIG. 4. SEM images of BNFA- $\mathrm{Cu} / \mathrm{Nb}$ samples. (a) The original (unpainted) image from Fig. 1(a). (b) Closeup view of the junction.

after precleaning (sputter etching) in the deposition chamber. The precleaning was used to remove eventual oxides at the surface of the first layer, thus obviating formation of weak links between the first and the second $\mathrm{Nb}$ layers. The total thickness of the $\mathrm{Nb}$ film was $\sim 200(70+130) \mathrm{nm}$. After this electrodes across the line were patterned by photolithography and reactive ion etching. During this etching all $\mathrm{Nb}$ and $\mathrm{Cu}$ layers unprotected by the photoresist mask were removed (also within the window line). Thus, six JJs (J1-J6) in each sample were made at the overlap between the window line and the crossing top electrodes, as sketched in Fig. 1(b). Junction sizes were $\sim 6 \times 5 \mu \mathrm{m}^{2}$ for the BNFA-Nb sample and $\sim 3 \times 5 \mu \mathrm{m}^{2}$ for the BNFA-Cu/Nb sample. Figures 4 and 5 (a) show SEM images of the studied samples. Each JJ has two electrodes, facilitating four-probe measurements.

\section{APPENDIX B: SAMPLE CHARACTERIZATION}

Measurements were performed in a dry ${ }^{3} \mathrm{He}$ cryostat in the temperature range $0.3 \mathrm{~K}<T<300 \mathrm{~K}$. The magnetic field, parallel to the junction plane, was supplied by the superconducting coil. The sample on the sample holder is shown in Fig. 5(b).

In-plane characteristics of BNFA were measured in the four-probe configuration by sending current through the outermost electrodes and measuring the voltage through the two inner electrodes [see Fig. 5(a)] (for details see Ref. [19]). Out-of-plane junction characteristics were measured in a quasi-four-probe configuration by sending current through one of the junction electrodes and measuring the voltage via another electrode on the same junction [see Fig. 5(a)] (for details see Ref. [18]).

Figure 5(c) shows $I_{c}(H)$ modulation patterns for the all BNFA- $\mathrm{Cu} / \mathrm{Nb}$ junctions at $3.6 \mathrm{~K}$. It is shown that all junctions exhibit clean Fraunhofer modulation, indicating good junction quality.

Figure 5(d) represents measurements of positive and negative critical currents versus the in-plane magnetic field. A slight tilt between the peak of the positive and the peak of the negative $I_{c}$ indicates the presence of a small self-field from the bias current [1], which slightly depends on the bias configuration. Minima/maxima of the Fraunhofer $I_{c}(H)$ pattern correspond to the integer/half-integer numbers of flux quanta in the junction. The inset in Fig. 5(d) shows the magnetic field of the maxima and minima versus the flux in the junction. The linearity indicates good periodicity of the $I_{c}(H)$ modulation and allows unambiguous extraction of the period, which is listed in Tables I and II. 

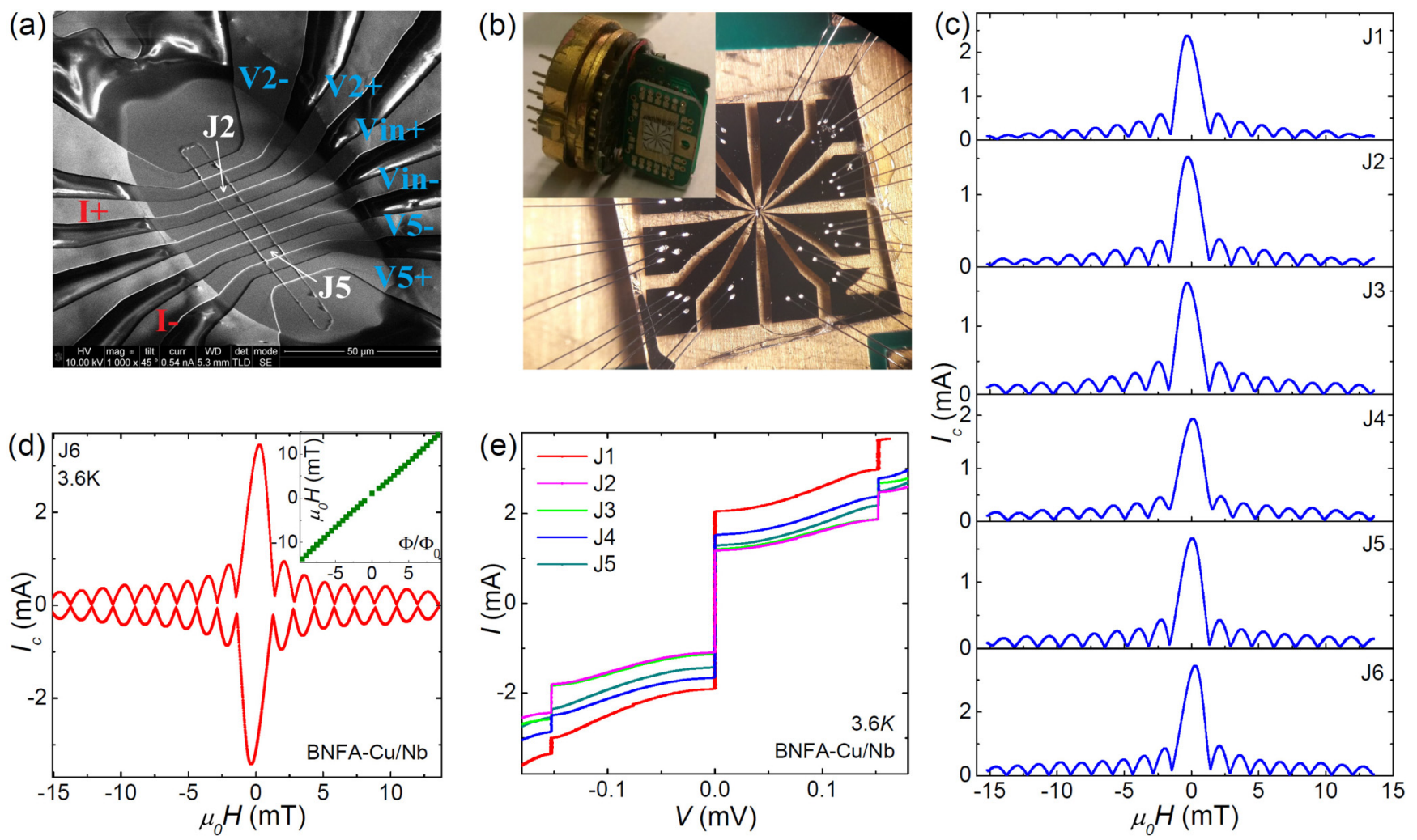

FIG. 5. BNFA sample characterization. (a) SEM image of a BNFA-Nb sample with a typical multiterminal configuration for in-plane (Vin+ Vin-) and junction $\mathrm{J} 2$ and $\mathrm{J} 5$ (V2+, V2 - and V5-, V5+) measurement. I+ and I- are current connections. (b) A sapphire (5×5 mm) substrate with a BNFA crystal and electrodes is glued and bonded to PCB. Inset: Sample holder with PCB. (c) Fraunhofer modulation $I_{c}(H)$ for all BNFA-Nb/Cu junctions at $3.6 \mathrm{~K}$. (d) The Fraunhofer modulation of positive and negative critical currents $I_{c}(H)$. Inset: Magnetic field of the maximum and the minimum positions. (e) $I-V$ curves of BNFA-Nb/Cu junctions $\mathrm{J} 1-\mathrm{J} 5$ in a microwave field (73.6 GHz).

Figure 5(e) shows $I-V$ curves of junctions in the BNFA$\mathrm{Cu} / \mathrm{Nb}$ sample with applied microwave radiation at $T=$ 3.6 K. Similar behavior with well-defined Shapiro steps is clearly shown. The comparison made in Figs. 5(c) and 5(e) demonstrates the consistency and reproducibility of junction characteristics, which allow material analysis of junction electrodes.

\section{APPENDIX C: SUMMARY OF JUNCTION PARAMETERS}

Table I and Table II summarize the characteristics of all junctions in BNFA-Nb and BNFA- $\mathrm{Cu} / \mathrm{Nb}$ samples. In the tables, $L_{x} \times L_{y}$ are the sizes of junctions; $S$ values are junction areas; $\mu_{0} \Delta H$ and $\mu_{0} \Delta H L_{x}$ are the period and normalized period of Fraunhofer modulation; $I_{c}$ and $J_{c}$ are the critical currents and critical current densities of the junctions; $R_{n}$ are normal resistances; and $R_{n} S$ is the junction resistance per square. The bottom line indicates the average values for $\mu_{0} \Delta H, \mu_{0} \Delta H L_{x}, J_{c}, R_{n} S$, and $I_{c} R_{n}$. The spread of junction parameters, $\sim 10 \%$ can be partly attributed to an uncertainty in determination of junction sizes and to a systematic reduction of the switching current by thermal fluctuations in smaller junctions. Overall there is good reproducibility of the fabrication process.

\section{APPENDIX D: DENSITY OF STATES FOR POLYCRYSTALLINE $\mathrm{Cu}$ and $\mathrm{Nb}$}

To clarify the difference in momentum selectivity of tunneling into polycrystalline $\mathrm{Cu}$ and $\mathrm{Nb}$, in Fig. 6 we show

TABLE I. Summary of characteristics of all measured junction BNFA-Nb samples at 3.6 K.

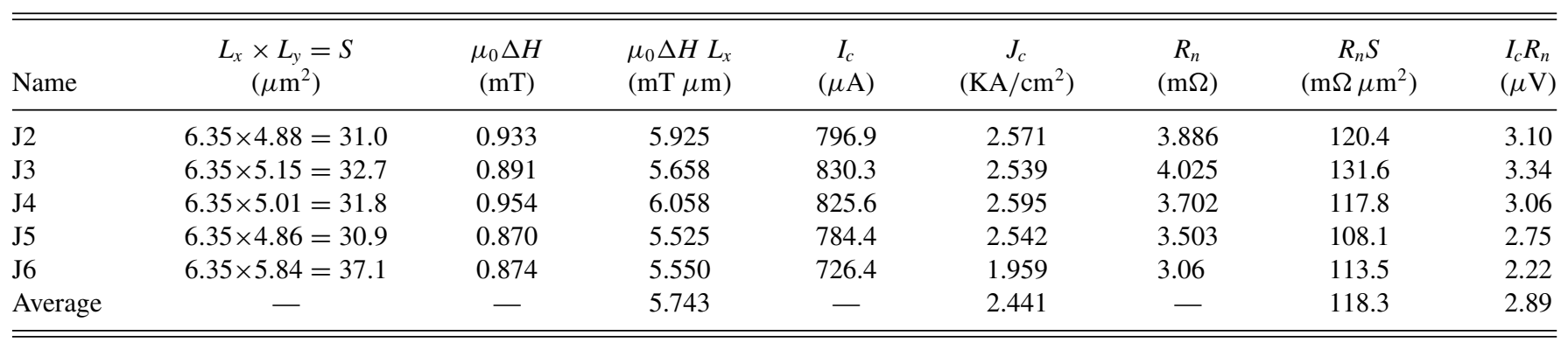


TABLE II. Summary of characteristics of all measured junction BNFA-Cu/Nb samples at $3.6 \mathrm{~K}$.

\begin{tabular}{|c|c|c|c|c|c|c|c|c|}
\hline $\mathrm{J} 1$ & $3.16 \times 6.09=19.2$ & 1.622 & 5.127 & 2266 & 11.78 & 58.49 & 1125 & 132.5 \\
\hline $\mathrm{J} 3$ & $2.90 \times 5.26=15.3$ & 1.732 & 5.026 & 1543 & 10.11 & 80.59 & 1230 & 124.4 \\
\hline $\mathrm{J} 4$ & $2.90 \times 5.21=15.1$ & 1.730 & 5.019 & 1834 & 12.14 & 71.21 & 1076 & 130.6 \\
\hline J5 & $2.98 \times 4.92=14.7$ & 1.654 & 4.929 & 1444 & 9.850 & 76.38 & 1120 & 110.3 \\
\hline Average & - & - & 5.019 & - & 11.56 & - & 1165 & 134.2 \\
\hline
\end{tabular}

cross-section profiles of DoS from Figs. 3(f) and 3(g). It is shown that $\mathrm{Cu}$ has a sharp peak in the DoS close to the edge and low in the center of the BZ. This leads to the enhancement of tunneling from $\mathrm{Cu}$ to $\mathrm{FS}$ sheets at the BZ corners and suppresses tunneling in the central FS sheets of the monocrystalline BNFA. For $\mathrm{Nb}$ the distribution of $\mathrm{DoS}$ is more even and both types of FS sheets participate in tunneling.

\section{APPENDIX E: NUMERICAL MODELING OF $I_{c} R_{n}(T)$ FOR JUNCTIONS WITH TWO-BAND SUPERCONDUCTORS}

Here we introduce a simple model for calculation of $I_{c} R_{n}$ for junctions between a two-band superconductor and a conventional $s$-wave superconductor $(\mathrm{Nb})$. It helps to clarify the broad-range variation of temperature dependencies of $I_{c} R_{n}$ in $s-s_{ \pm}$junctions.

Cooper pair tunneling between conventional $s$-wave superconductors is described by the Ambegaokar-Baratoff model [4],

$$
I_{c} R_{n}=\frac{\pi k_{B} T}{e} \Delta_{l} \Delta_{r} \sum_{n=-\infty}^{+\infty}\left[\left(\omega_{n}^{2}+\Delta_{l}^{2}\right)\left(\omega_{n}^{2}+\Delta_{r}^{2}\right)\right]^{-1 / 2},
$$

where $n$ is an integer, $\omega_{n}=2 \pi k_{B} T(2 n+1)$, and $\Delta_{l, r}$ are temperature-dependent energy gaps in the left and right electrodes of the JJ. This expression is obtained assuming specular transmission with a constant, energy-independent tunneling matrix element. As mentioned in Sec. I, all band-structure effects are encoded in the $R_{n}$ value and the expression, Eq. (E1), for $I_{c} R_{n}$ is universal (band structure independent). For a

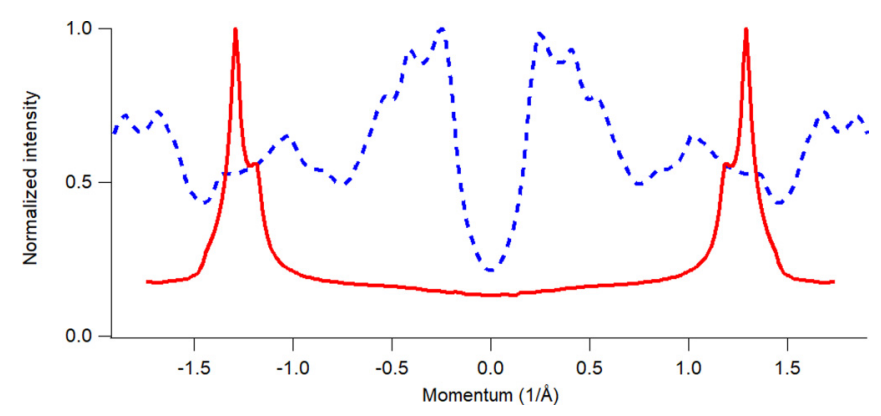

FIG. 6. Radial momentum distributions of $k_{z}$-integrated projections of Fermi surfaces on the (001) plane for $\mathrm{Cu}$ (solid red curve) and $\mathrm{Nb}$ (dashed blue curve) averaged over the in-plane momentum angle. These profiles represent the effective density-of-states distribution for polycrystalline $\mathrm{Cu}$ and $\mathrm{Nb}$ films. junction with identical superconductors, $\Delta_{l}=\Delta_{r}=\Delta$, $I_{c} R_{n}(T=0)=(\pi / 2 e) \Delta(T=0)$, where $e$ is the electron charge.

For the considered JJ, the corresponding energy gaps are $\Delta$ in the $s$ electrode and $\Delta_{1}$ and $\Delta_{2}$ for the two-band superconductor. In the trivial $s_{++}$case, the gaps have the same sign, $\operatorname{sign}\left(\Delta_{1}\right)=\operatorname{sign}\left(\Delta_{2}\right)$. In the nontrivial sign-reversal $s_{ \pm}$ case, $\operatorname{sign}\left(\Delta_{1}\right)=-\operatorname{sign}\left(\Delta_{2}\right)$. For modeling of our junctions, we take the slightly reduced value of $\Delta(0)=1.3 \mathrm{meV}$ for $\mathrm{Nb}$, which is relevant for the proximity-induced $\Delta$ in the $\mathrm{Cu}-\mathrm{Nb}$ bilayer [38]. For BNFA we assume the values $\Delta_{1}=7.8 \mathrm{meV}$ and $\left|\Delta_{2}\right|=2.3 \mathrm{meV}$, deduced from ARPES data [39]. Although these gaps are known only approximately and values in a fairly broad range, 5-15 meV for $\Delta_{1}$ and $1.5-5 \mathrm{meV}$ for $\left|\Delta_{2}\right|$, can be found in the literature [40-44], qualitative results, described below, do not depend on exact values of $\Delta_{1,2}$ and remain valid for any combination $\Delta_{1}>\left|\Delta_{2}\right|>\Delta$.

Figure 7(a) shows the temperature dependencies of the gaps for Nb-BNFA JJs. We assume that all of them follow the standard BCS $T$ dependence. Due to the significant difference in critical temperatures of $\mathrm{Nb}(8.5 \mathrm{~K})$ and BNFA $(30 \mathrm{~K}), \Delta_{1,2}$ are practically constant in the range $T<T_{c}(\mathrm{Nb})$, as indicated by the thick portions of the corresponding lines for $\Delta_{1,2}(T)$ in Fig. 7(a).

We assume that tunneling into each of the two bands of the pnictide occurs independently and is described by Eq. (E1) with corresponding $I_{c 1} R_{n 1}$ and $I_{c 2} R_{n 2}$ values. The temperature dependencies of these, obtained for $\Delta(T)$ and $\Delta_{1,2}(T)$ in Fig. 7(a), are shown by blue and red lines in Fig. 7(b) along with $I_{c} R_{n}(T)$ for a symmetric $s-s$ Nb-Nb JJ (black line). The difference in $T$ dependencies is easily understood. For a symmetric Nb-Nb JJ, close to $T_{c}(\mathrm{Nb}) I_{c} R_{n} \propto \Delta^{2}(T)$ is linear, $\propto 1-T / T_{c}$. For an asymmetric JJ $\Delta_{1} \gg \Delta$ with constant $\Delta_{1}(T), I_{c 1} R_{n 1} \propto \Delta(T)$, as can be seen from the practically identical shapes of the blue lines in Figs. 7(a) and 7(b). Therefore, $I_{c 1} R_{n 1}$ has a convex shape with a sharp drop $\propto\left(1-T / T_{c}\right)^{0.5}$ close to $T_{c}(\mathrm{Nb})$. The temperature dependence of $\left|I_{c 2} R_{n 2}\right|$ with a significantly smaller $\left|\Delta_{2}\right| \ll \Delta_{1}$ is between these two limits and has a slightly softer convex shape at $T \sim T_{c}(\mathrm{Nb})$. The slight difference in the shapes of $I_{c 1} R_{n 1}(T)$ and $\left|I_{c 2} R_{n 2}(T)\right|$ is important in further analysis.

Since the two currents flow in parallel, the total supercurrent is $I_{c}=I_{c 1}+I_{c 2}$, the total resistance $R_{n}=R_{n 1} R_{n 2} /$ $\left(R_{n 1}+R_{n 2}\right)$, and

$$
I_{c} R_{n}=I_{c 1} R_{n 1} \frac{1}{1+\alpha}+I_{c 2} R_{n 2} \frac{\alpha}{1+\alpha},
$$



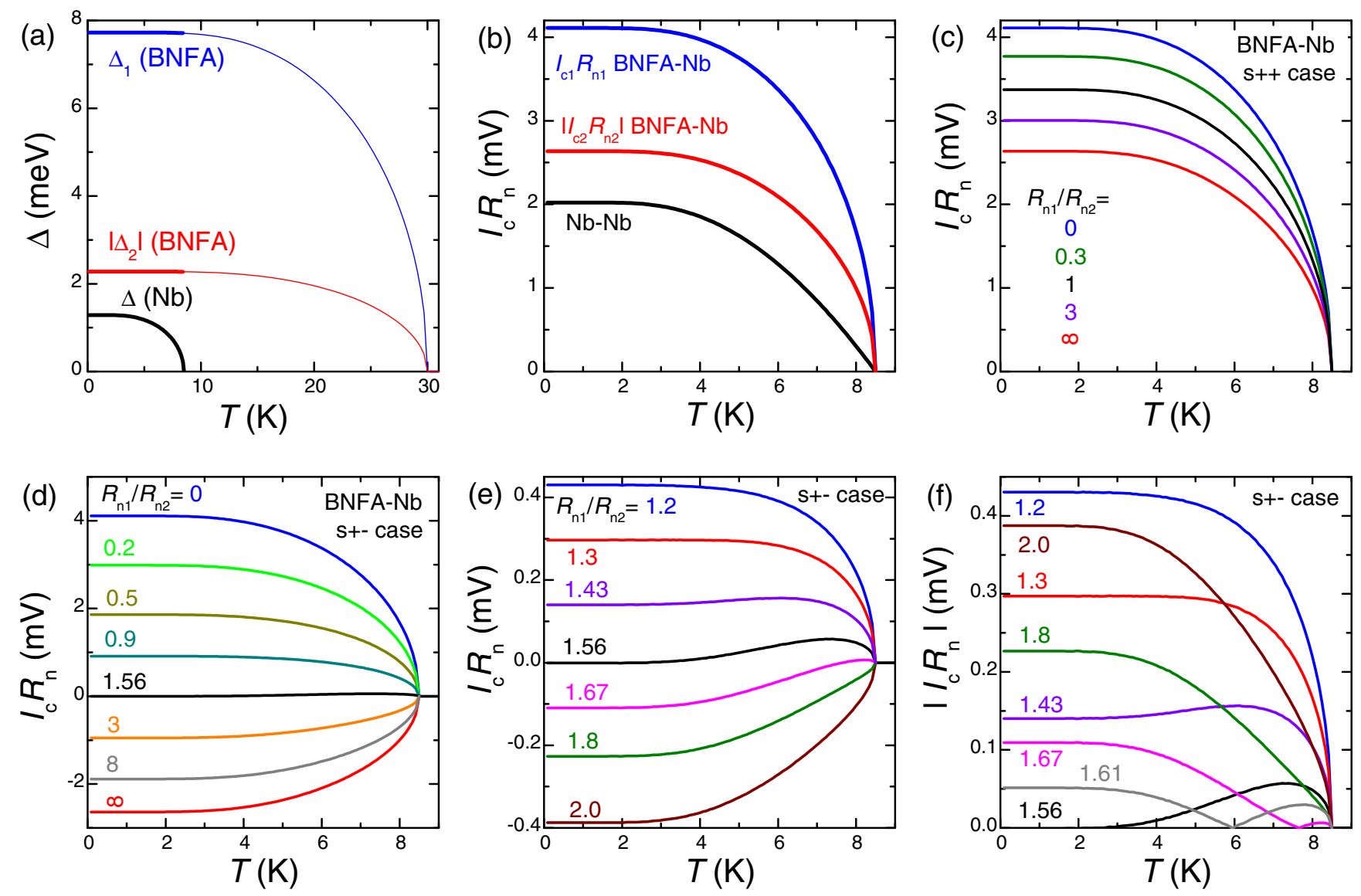

FIG. 7. Numerical simulation of $I_{c} R_{n}$ in Nb-BNFA junctions. (a) Temperature dependencies of energy gaps. (b) Blue and red lines represent $I_{c 1} R_{n 1}$ and $I_{c 2} R_{n 2}$ for tunneling from $s$ in each of the two bands of BNFA. For comparison, the black line shows $I_{c} R_{n}$ for a symmetric $s-s$ Nb-Nb junction. (c), (d) Temperature dependencies $I_{c} R_{n}(T)$ for different relative transparencies of the two channels, $\alpha=R_{n 1} / R_{n 2} \in[0, \infty]$, for sign-conserving $s-s_{++}$(c) and sign-reversal $s-s_{ \pm}$(d) junctions. (e) $I_{c} R_{n}(T)$ for different $R_{n 1} / R_{n 2}$ values in sign-reversal $s-s_{ \pm}$junctions close to the cancellation point. (f) Experimentally measurable absolute values of $\left|I_{c} R_{n}(T)\right|$ for the $s-s_{ \pm}$case close to the cancellation point. Note that dramatic amplitude and shape variations of $I_{c} R_{n}(T)$ occur only in the sign-reversal $s_{ \pm}$cases (d)-(f) and do not happen in the sign-conserving $s_{++}$case (c).

where $\alpha=R_{n 1} / R_{n 2}$. Thus the $I_{c} R_{n}$ depends on only one parameter, $\alpha$, which represents the relative transparencies of the two channels: $\alpha=0$ corresponds to Cooper pair tunneling only in the first channel $\left(\Delta_{1}\right)$ and $\alpha=\infty$ only in the second channel $\left(\Delta_{2}\right)$. For an intermediate $\alpha$ both channels contribute to $I_{c} R_{n}$.

First, we consider the trivial sign-conserving $s_{++}$case. A gradual transformation of $I_{c} R_{n}(T)$ with variation of $\alpha$ from 0 to $\infty$ in this case is shown in Fig. 7(c). It is shown that the value of $I_{c} R_{n}(0)$ changes only modestly, from 4.1 to $2.6 \mathrm{mV}$, and the shape of $I_{c} R_{n}(T)$ changes only slightly. Therefore, for the $s-s_{++} \mathrm{JJ}$, the FS geometry effect, although not negligible, remains small. It certainly does not explain the observed two orders of magnitude variation of $I_{c} R_{n}$ for the two types of studied JJs.

Next we consider the $s-s_{ \pm} \mathrm{JJ}$ with a sign-reversal superconductor. In this case $I_{c 1}$ and $I_{c 2}$ have opposite signs. The choice of signs does not matter; we assume $I_{c 1}>0$ and $I_{c 2}<0$. Figure 7(d) shows the variation of $I_{c} R_{n}(T)$ with a changing $\alpha$ in this case. Here $I_{c} R_{n}(0)$ changes from $I_{c 1} R_{n 1}(0) \simeq+4.1$ to $I_{c 2} R_{n 2}(0) \simeq-2.6 \mathrm{mV}$. For finite $\alpha$ both channels contribute and partly cancel each other. The complete cancellation, $I_{c 1}=-I_{c 2}$, occurs at $\alpha=I_{c 1} R_{n 1} /\left|I_{c 2} R_{n 2}\right|$.
Figure 7(e) represents the calculated temperature dependencies of $I_{c} R_{n}$ close to the cancellation point, when $I_{c} R_{n} \ll$ $\max \left(\left|\Delta_{1,2} / e\right|\right)$. For the chosen gaps, complete cancellation of $I_{c}(T=0)$ occurs at $\alpha \simeq 1.56$ (black line). However, due to the difference in the shapes of $I_{c 1} R_{n 1}(T)$ and $I_{c 2} R_{n 2}(T)$, the ratio $I_{c 1} R_{n 1} / I_{c 2} R_{n 2}$ is temperature dependent. Therefore, there is no complete cancellation of $I_{c}$ at all $T$ and the critical current recovers at elevated temperatures. This leads to a profound nonmonotonous temperature dependence of $I_{c} R_{n}$ close to the cancellation point, qualitatively consistent with an earlier result [7]. In Fig. 7(c) it is also shown that the shape of $I_{c} R_{n}(T)$ is different on the positive and negative sides $(\pi$-junction case). On the positive side (see the curves for $\alpha=1.2$ and 1.3), $I_{c} R_{n}(T)$ has a convex shape close to $T_{c}$. On the negative side it first becomes concave (see the curve at $\alpha=1.67$ ), then linear at $\alpha=1.8$ and slightly convex close to $T_{c}$ at larger $\alpha=$ 2 . The described variation of the $I_{c} R_{n}(T)$ shape is qualitatively consistent with results from Ref. [8], although calculations in that work were done for in-plane JJs, different from our JJs with an out-of-plane ( $c$-axis) orientation.

Experimental measurements of the critical current reveal only the absolute value of $I_{c}$. Therefore, for comparison with experiment in Fig. 7(f) we show the absolute values of $I_{c} R_{n}(T)$ 
in the $s-s_{ \pm}$case close to the cancellation point. One can see spectacular variation of both the amplitudes, $I_{c} R_{n}(0)$, and the shapes of $I_{c} R_{n}(T)$ curves. Even the nonmonotonous dependence of $I_{c} R_{n}(T)$ can be observed due to the transition between 0 and $\pi$ states as in SFS JJs [45]. This is in stark contrast with the behavior of $s-s_{++}$junctions [see Fig. 7(c)], which have only a marginal dependence on $\alpha$ and, thus, on the electronic structure and the FS geometry. Simulations in Fig. 7(f) confirm our statements that FS geometries play a crucial role for JJs with sign-reversal multiband superconductors. This does not happen in the sign-conserving $s-s_{++}$case (at any junction parameters).

Thus, the observation of a strong dependence of $I_{c} R_{n}$ on the material of the thin $\mathrm{N}$ interlayer is a consequence of the sign-reversal $s_{ \pm}$order in the pnictide. As discussed above, it originates from the strong FS-geometry sensitivity of the Josephson effect in $s-s_{ \pm}$JJs close to the cancellation point. At the cancellation point the FS sensitivity is infinite because $I_{c}$ changes from 0 to a finite value. Presented data are consistent with this scenario: BNFA-Nb JJs with $I_{c} R_{n}(0) \simeq 3 \mu \mathrm{V} \ll$
$\left|\Delta_{1,2}\right| / e$ are very close to the cancellation point. This implies that tunneling into both bands of BNFA occurs at similar rates, $I_{c 1} \simeq-I_{c 2}$. This is consistent with the FS-geometry analysis, shown in Fig. 3(i). Insertion of a thin $\mathrm{Cu}$ interlayer with a different FS geometry partly blocks tunneling into the central band, as shown in Fig. 3(h), which removes the cancellation. As follows from simulations in Fig. 7(f), even a very small, $\lesssim 10 \%$, change in the relative channel transparencies, $\alpha$, in this case (from 1.56 to 1.67 or 1.43 ) easily explains the almost two order of magnitude enhancement of $I_{c} R_{n}$ in BNFA-Cu/Nb JJs. Moreover, while near-cancellation of $I_{c} R_{n}$ in BNFA-Nb junctions provides evidence of the $s_{ \pm}$symmetry in BNFA, the dramatic enhancement of $I_{c} R_{n}$ in NBFA-Cu/Nb junctions points out that the sign reversal occurs between central and corner bands of BNFA. This, although anticipated [12-15], has not yet been proven experimentally. Therefore, as emphasized in the text, the performed analysis of the material (FS-geometry) dependence of the Josephson effect provides a new tool for phase-sensitive studies and paves the way to conscious FS engineering of pnictide-based junctions.
[1] A. Barone and C. Paterno, Physics and Applications of the Josephson Effect (Wiley, New York, 1982).

[2] K. K. Likharev, Dynamics of Josephson Junctions and Circuits (Gordon and Breach Science, Amsterdam, 1986).

[3] D. G. McDonald, The Nobel laureate versus the graduate student, Phys. Today 54(7), 46 (2001).

[4] V. Ambegaokar and A. Baratoff, Tunneling between Superconductors, Phys. Rev. Lett. 10, 486 (1963); Erratum: 11, 104 (1963).

[5] Y. Tanaka and S. Kashiwaya, Theory of Josephson effects in anisotropic superconductors, Phys. Rev. B 56, 892 (1997).

[6] Y. Ota, M. Machida, T. Koyama, and H. Matsumoto, Theory of Heterotic Superconductor-Insulator-Superconductor Josephson Junctions between Single- and Multiple-Gap Superconductors, Phys. Rev. Lett. 102, 237003 (2009).

[7] I. B. Sperstad, J. Linder, and A. Sudbø, Quantum transport in ballistic $s_{ \pm}$-wave superconductors with interband coupling: Conductance spectra, crossed Andreev reflection, and Josephson current, Phys. Rev. B 80, 144507 (2009).

[8] A. V. Burmistrova, I. A. Devyatov, A. A. Golubov, K. Yada, Y. Tanaka, M. Tortello, R. S. Gonnelli, V. A. Stepanov, X. Ding, H. H. Wen, and L. H. Green, Josephson current in Fe-based superconducting junctions: Theory and experiment, Phys. Rev. B 91, 214501 (2015).

[9] D. A. Wollman, D. J. Van Harlingen, W. C. Lee, D. M. Ginsberg, and A. J. Leggett, Experimental Determination of the Superconducting Pairing State in YBCO from the Phase Coherence of YBCO-Pb DC SQUIDs, Phys. Rev. Lett. 71, 2134 (1993).

[10] C. C. Tsuei and J. R. Kirtley, Pairing symmetry in cuprate superconductors, Rev. Mod. Phys. 72, 969 (2000).

[11] T. K. Ng and N. Nagaosa, Broken time-reversal symmetry in Josephson junction involving two-band superconductors, Europhys. Lett. 87, 17003 (2009).

[12] C.-T. Chen, C. C. Tsuei, M. B. Ketchen, Z.-A. Ren, and Z. X. Zhao, Integer and half-integer flux-quantum transitions in a niobium-iron pnictide loop, Nat. Phys. 6, 260 (2010).
[13] P. J. Hirschfeld, M. M. Korshunov, and I. I. Mazin, Gap symmetry and structure of Fe-based superconductors, Rep. Prog. Phys. 74, 124508 (2011).

[14] F. Wang and D.-H. Lee, The electron-pairing mechanism of iron-based superconductors, Science 332, 200 (2011).

[15] A. Chubukov, Pairing mechanism in Fe-based superconductors, Annu. Rev. Condens. Matter Phys. 3, 57 (2012).

[16] T. Hanaguri, S. Niitaka, K. Kuroki, and H. Takagi, Unconventional $s$-wave superconductivity in $\mathrm{Fe}(\mathrm{Se}, \mathrm{Te})$, Science 328, 474 (2010).

[17] J.-X. Yin, S. S. Zhang, G. Dai, Y. Zhao, A. Kreisel, G. Macam, X. Wu, H. Miao, Z. Q. Huang, J. H. J. Martiny, B. M. Andersen, N. Shumiya, D. Multer, M. Litskevich, Z. Cheng, X. Yang, T. A. Cochran, G. Chang, I. Belopolski, L. Xing, X. Wang, Y. Gao, F. C. Chuang, H. Lin, Z. Wang, C. Jin, Y. Bang, and M. Z. Hasan, Quantum Phase Transition of Correlated Iron-Based Superconductivity in $\mathrm{LiFe}_{1-x} \mathrm{Co}_{x}$ As, Phys. Rev. Lett. 123, 217004 (2019).

[18] A. A. Kalenyuk, A. Pagliero, E. A. Borodianskyi, A. A. Kordyuk, and V. M. Krasnov, Phase-Sensitive Evidence for the Sign-Reversal $s_{ \pm}$Symmetry of the Order Parameter in an Iron-Pnictide Superconductor using $\mathrm{Nb} / \mathrm{Ba}_{1-x} \mathrm{Na}_{x} \mathrm{Fe}_{2} \mathrm{As}_{2}$ Josephson Junctions, Phys. Rev. Lett. 120, 067001 (2018).

[19] A. A. Kalenyuk, A. Pagliero, E. A. Borodianskyi, S. Aswartham, S. Wurmehl, B. Büchner, D. A. Chareev, A. A. Kordyuk, and V. M. Krasnov, Unusual two-dimensional behavior of iron-based superconductors with low anisotropy, Phys. Rev. B 96, 134512 (2017).

[20] Y. Liu, M. A. Tanatar, W. E. Straszheim, B. Jensen, K. W. Dennis, R. W. McCallum, V. G. Kogan, R. Prozorov, and T. A. Lograsso, Comprehensive scenario for single-crystal growth and doping dependence of resistivity and anisotropic upper critical fields in $\left(\mathrm{Ba}_{1-x} \mathrm{~K}_{x}\right) \mathrm{Fe}_{2} \mathrm{As}_{2}(0.22 \leqslant x \leqslant 1)$, Phys. Rev. B 89, 134504 (2014).

[21] A. Zeinali, T. Golod, and V. M. Krasnov, Surface superconductivity as the primary cause of broadening of superconducting 
transition in $\mathrm{Nb}$ films at high magnetic fields, Phys. Rev. B 94, 214506 (2016).

[22] S. O. Katterwe, A. Rydh, and V. M. Krasnov, DopingInduced Change in the Interlayer Transport Mechanism of $\mathrm{Bi}_{2} \mathrm{Sr}_{2} \mathrm{CaCu}_{2} \mathrm{O}_{8+\delta}$ Near the Superconducting Transition Temperature, Phys. Rev. Lett. 101, 087003 (2008).

[23] A. A. Golubov, M. Yu. Kupriyanov, and E. Il'ichev, The current-phase relation in Josephson junctions, Rev. Mod. Phys. 76, 411 (2004).

[24] V. M. Krasnov, N. F. Pedersen, V. A. Oboznov, and V. V. Ryazanov, Josephson properties of $\mathrm{Nb} / \mathrm{Cu}$ multilayers, Phys. Rev. B 49, 12969 (1994).

[25] V. M. Krasnov, V. A. Oboznov and V. V. Ryazanov, Anomalous temperature dependence of $H_{c 1}^{\perp}$ in superconducting $\mathrm{Nb} / \mathrm{Cu}$ multilayer, Physica C 196, 335 (1992).

[26] A. A. Golubov, E. P. Houwman, J. G. Gijsbertsen, V. M. Krasnov, J. Flokstra, and H. Rogalla, Proximity effect in superconductor-insulator-superconductor Josephson tunnel junctions: Theory and experiment, Phys. Rev. B 51, 1073 (1995).

[27] http://www.phys.ufl.edu/fermisurface/.

[28] T.-S. Choy, J. Naset, J. Chen, S. Hershfield, and C. Stanton, A database of Fermi surface in virtual reality modeling language (VRML), Bull. Am. Phys. Soc. 45, L36.042 (2000).

[29] A. N. Yaresko (private communication).

[30] V. B. Zabolotnyy, D. S. Inosov, D. V. Evtushinsky, A. Koitzsch, A. A. Kordyuk, G. L. Sun, J. T. Park, D. Haug, V. Hinkov, A. V. Boris, C. T. Lin, M. Knupfer, A. N. Yaresko, B. Büchner, A. Varykhalov, R. Follath, and S. V. Borisenko, $(\pi, \pi)$ electronic order in iron arsenide superconductors, Nature 457, 569 (2009).

[31] A. A. Kordyuk, V. B. Zabolotnyy, D. V. Evtushinsky, A. N. Yaresko, B. Büchner, and S. V. Borisenko, Electronic band structure of ferro-pnictide superconductors from ARPES experiment, J. Supercond. Nov. Magn. 26, 2837 (2013).

[32] S. Döring, D. Reifert, N. Hasan, S. Schmidt, F. Schmidl, V. Tympel, F. Kurth, K. Iida, B. Holzapfel, T. Wolf, and P. Seidel, Excess currents in planar $\mathrm{Ba}\left(\mathrm{Fe}_{1-x} \mathrm{Co}_{x}\right)_{2} \mathrm{As}_{2} / \mathrm{TiO}_{x} / \mathrm{Pb}$ Josephson junctions, Phys. Status Solidi B 252, 2858 (2015).

[33] S. Schmidt, S. Döring, N. Hasan, F. Schmidl, V. Tympel, F. Kurth, K. Iida, H. Ikuta, T. Wolf, and P. Seidel, Josephson effects at iron pnictide superconductors: Approaching phasesensitive experiments, Phys. Status Solidi B 254, 1600165 (2017).

[34] W. Tian, Y. Lv, Z. Xu, H. Zhang, S. Chen, S. Dong, J. Li, Y.-L. Wang, D. Kölle, R. Kleiner, H. Wang, and P. Wu, Hybrid $\mathrm{Nb} / \mathrm{Al} / \mathrm{Ba}_{0.5} \mathrm{~K}_{0.5} \mathrm{Fe}_{2} \mathrm{As}_{2}$ sandwich Josephson junctions, Supercond. Sci. Technol. 33, 025014 (2020).

[35] N. Barisic, S. Badoux, M. K. Chan, C. Dorow, W. Tabis, B. Vignolle, G. Yu, J. Beard, X. Zhao, C. Proust, and M. Greven,
Universal quantum oscillations in the underdoped cuprate superconductors, Nat. Phys. 9, 761 (2013).

[36] S. E. Sebastian, N. Harrison, F. F. Balakirev, M. M. Altarawneh, P. A. Goddard, R. Liang, D. A. Bonn, W. N. Hardy, and G. G. Lonzarich, Normal-state nodal electronic structure in underdoped high- $T_{c}$ copper oxides, Nature (London) 511, 61 (2014).

[37] V. M. Krasnov, Superconducting condensate residing on small Fermi pockets in underdoped cuprates, Phys. Rev. B 91, 224508 (2015).

[38] V. M. Krasnov, H. Motzkau, T. Golod, A. Rydh, S. O. Katterwe, and A. B. Kulakov, Comparative analysis of tunneling magnetoresistance in low- $T_{c} \mathrm{Nb} / \mathrm{Al}-\mathrm{AlOx} / \mathrm{Nb}$ and high- $T_{c} \mathrm{Bi}_{2-y} \mathrm{~Pb}_{y} \mathrm{Sr}_{2} \mathrm{CaCu}_{2} \mathrm{O}_{8+\delta}$ intrinsic Josephson junctions, Phys. Rev. B 84, 054516 (2011).

[39] D. V. Evtushinsky, V. B. Zabolotnyy, L. Harnagea, A. N. Yaresko, S. Thirupathaiah, A. A. Kordyuk, J. Maletz, S. Aswartham, S. Wurmehl, E. Rienks, R. Follath, B. Büchner, and S. V. Borisenko, Electronic band structure and momentum dependence of the superconducting gap in $\mathrm{Ca}_{1-x} \mathrm{Na}_{x} \mathrm{Fe}_{2} \mathrm{As}_{2}$ from angle-resolved photoemission spectroscopy, Phys. Rev. B 87, 094501 (2013).

[40] P. Szabó, Z. Pribulová, G. Pristáš, S. L. Budko, P. C. Canfield, and P. Samuely, Evidence for two-gap superconductivity in $\mathrm{Ba}_{0.55} \mathrm{~K}_{0.45} \mathrm{Fe}_{2} \mathrm{As}_{2}$ from directional point-contact Andreevreflection spectroscopy, Phys. Rev. B 79, 012503 (2009).

[41] D. Daghero, M. Tortello, G. A. Ummarino, and R. S. Gonnelli, Directional point-contact Andreev-reflection spectroscopy of Fe-based superconductors: Fermi surface topology, gap symmetry, and electron-boson interaction, Rep. Prog. Phys. 74, 124509 (2011).

[42] Yu. G. Naidyuk, O. E. Kvitnitskaya, S. Aswartham, G. Fuchs, K. Nenkov, and S. Wurmehl, Exploring point-contact spectra of $\mathrm{Ba}_{1-x} \mathrm{Na}_{x} \mathrm{Fe}_{2} \mathrm{As}_{2}$ in the normal and superconducting states, Phys. Rev. B 89, 104512 (2014).

[43] S. Ziemak, K. Kirshenbaum, S. R. Saha, R. Hu, J.-Ph. Reid, R. Gordon, L. Taillefer, D. Evtushinsky, S. Thirupathaiah, B. Büchner, S. V. Borisenko, A. Ignatov, D. Kolchmeyer, G. Blumberg, and J. Paglione, Isotropic multi-gap superconductivity in $\mathrm{BaFe}_{1} \cdot 9 \mathrm{Pt}_{0} \cdot 1 \mathrm{As}_{2}$ from thermal transport and spectroscopic measurements, Supercond. Sci. Technol. 28, 014004 (2015).

[44] Y. F. Wu, A. B. Yu, L. B. Lei, C. Zhang, T. Wang, Y. H. Ma, Z. Huang, L. X. Chen, Y. S. Liu, C. M. Schneider, G. Mu, H. Xiao, and $\mathrm{T}$. $\mathrm{Hu}$, Superconducting $\mathrm{NbN}$ and $\mathrm{CaFe}_{0.88} \mathrm{Co}_{0.12} \mathrm{AsF}$ studied by point-contact spectroscopy with a nanoparticle $\mathrm{Au}$ array, Phys. Rev. B 101, 174502 (2020).

[45] V. V. Ryazanov, V. A. Oboznov, A. Yu. Rusanov, A. V. Veretennikov, A. A. Golubov, and J. Aarts, Coupling of Two Superconductors Through a Ferromagnet: Evidence for a $\pi$ Junction, Phys. Rev. Lett. 86, 2427 (2001). 